\title{
Effects of birth weight on body composition and overweight/obesity at early school age
}

\section{Jing Zhou ${ }^{\mathrm{a}}$}

Lingxia Zeng

Duolao Wang ${ }^{c}$

Chao $\mathrm{Li}^{\mathrm{b}}$

Yuesheng Liu ${ }^{\mathrm{a}}$

Hong Yan ${ }^{\mathrm{b}, \mathrm{d}, \mathrm{e}}$

Yanfeng Xiao ${ }^{\mathrm{a}}$, *

xyf_xjtu@163.com

a Department of Pediatrics, The Second Affiliated Hospital of Xi'an Jiaotong University, Xi'an, Shaanxi, PR China

${ }^{\mathbf{b}}$ Department of Epidemiology and Biostatistics, School of Public Health, Health Science Center, Xi'an Jiaotong University, Xi'an, Shaanxi, PR China

${ }^{\mathbf{c}}$ Department of Clinic Science, Liverpool School of Tropical Medicine, Liverpool, United Kingdom

d Nutrition and Food Safety Engineering Research Center of Shaanxi Province, Xi'an, Shaanxi, PR China

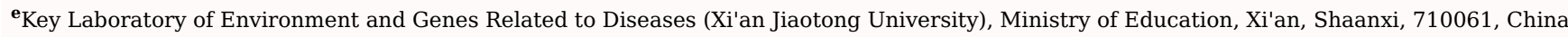

${ }^{*}$ Corresponding author. Department of Pediatrics, The Second Affiliated Hospital of Xi'an Jiaotong University, No. 157, Xi Wu Road, Xi'an, Shaanxi, 710004, PR China.

\section{Summary}

objectives

The prevalence of childhood obesity has increased substantially. We aimed to characterize the effect of birth weight on body composition and overweight/obesity at early school age. Study design

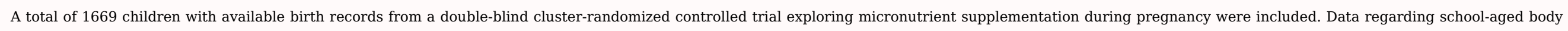
composition, social-demographic factors and health behaviours were prospectively collected.

\section{Result}

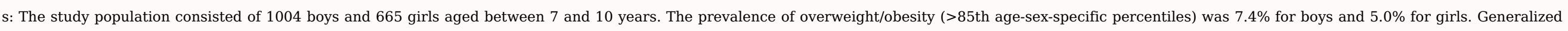

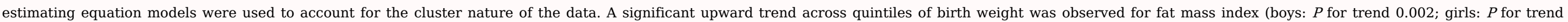

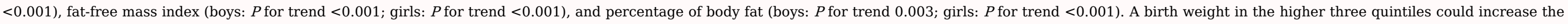

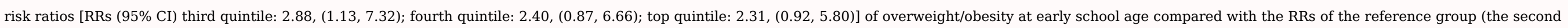
quintile of birth weight) among boys.

Conclusions

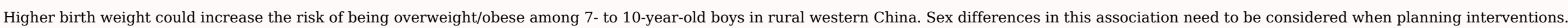

\section{Research registration}


Keywords: Birth weight; Obesity; Body composition; Early school-aged; Follow-up study

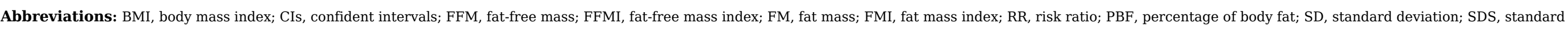
deviation score

\section{Introduction}

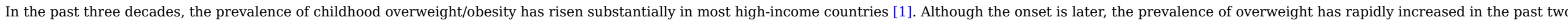

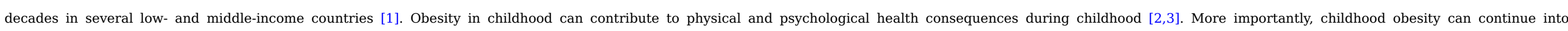
adolescence and adulthood, resulting in health and economic consequences [4,5].

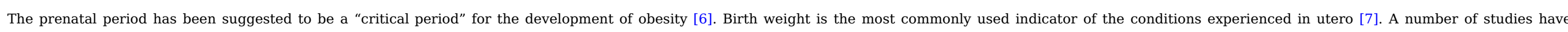

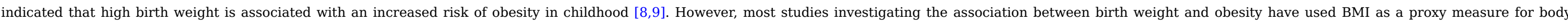

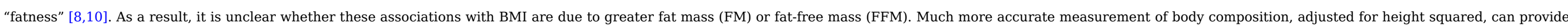
independent and more precise indicators of fatness, leanness, and adiposity among children [11].

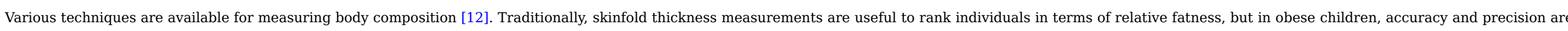

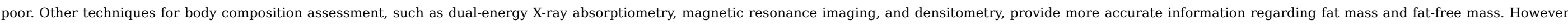

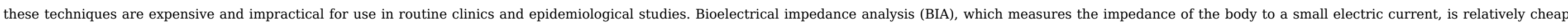
and easy to use. Therefore, at present BIA is primarily an epidemiological technique.

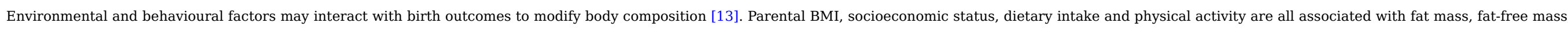

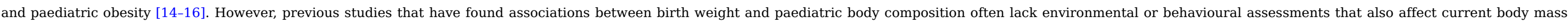

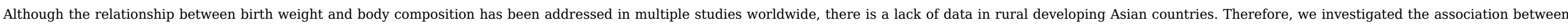

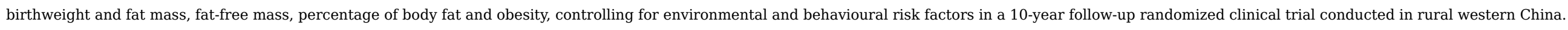

\section{Methods}

\subsection{Study design and participants}

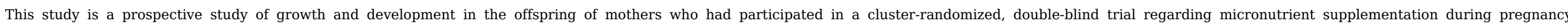

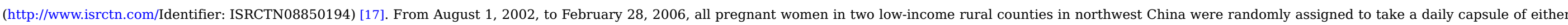

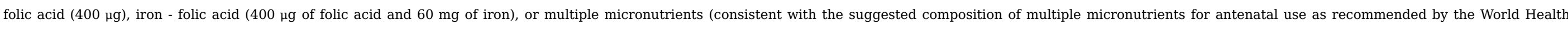
Organization [WHO]) [18].

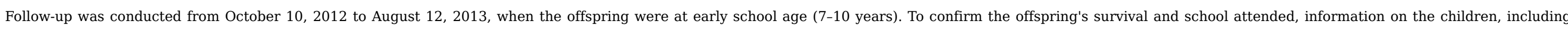

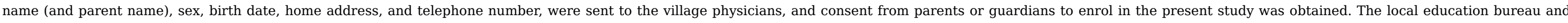

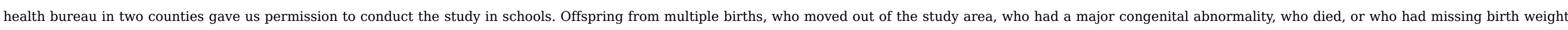

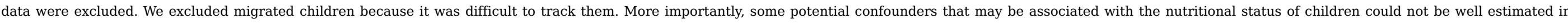
migrated children.

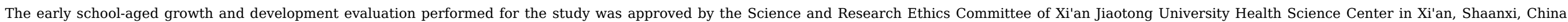




\subsection{Birth weight, body composition and overweight/obesity}

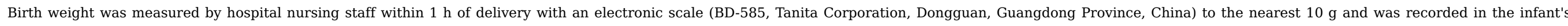

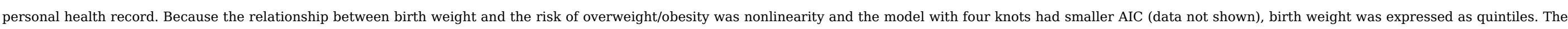

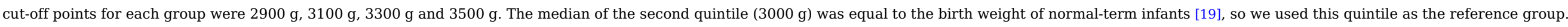

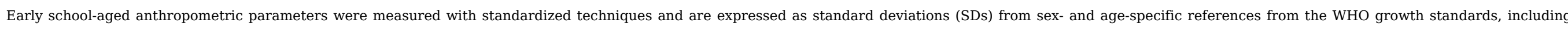

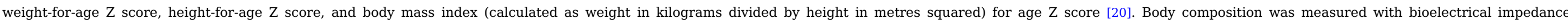

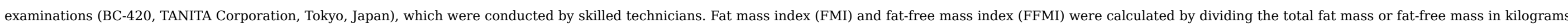

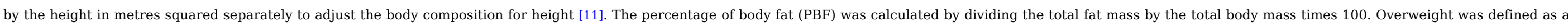
PBF between the 85th and 95th percentiles of each age and sex group, and obesity was defined as PBF above the 95th percentile of each age and sex group [21].

\subsection{Confounding variables}

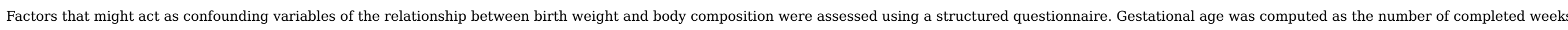

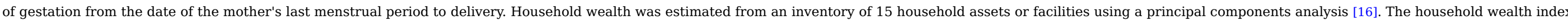
was categorized into tertiles, indicating the low-income, middle-income, and highest-income households. Measured parental height and weight were used to calculate parental BMI

\subsection{Statistical analysis}

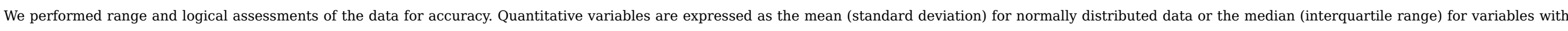

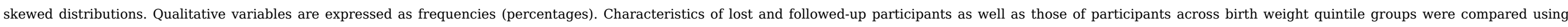
analyses of variance (normally distributed data) or Mann-Whitney $U$ tests (abnormally distributed data) and $\chi^{2}$ tests.

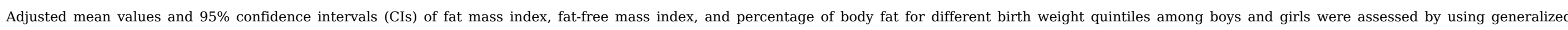

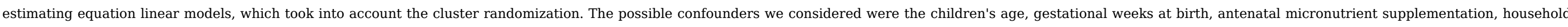

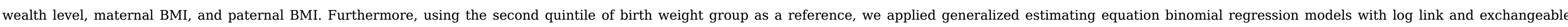

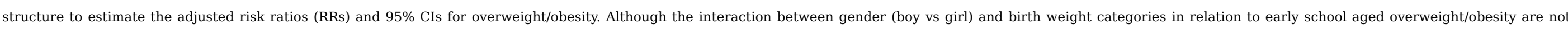

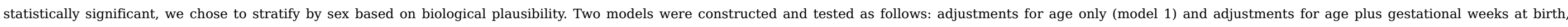

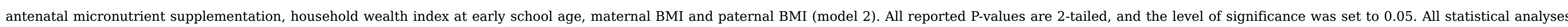
were conducted using SPSS version 20.0 for PC (International Business Machines Corporation, Armonk, New York, United States).

\section{Results}

\subsection{Followed participants}

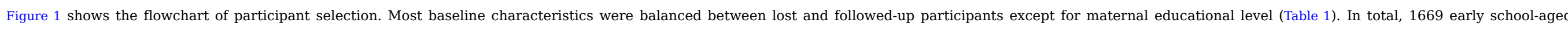

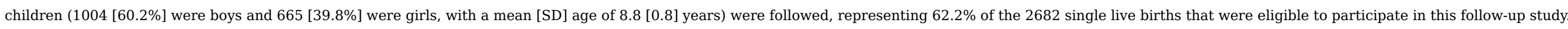




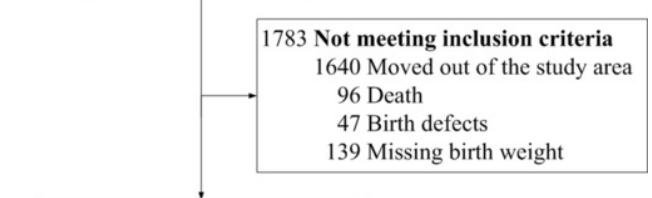

\section{Enrolled in follow-up study}

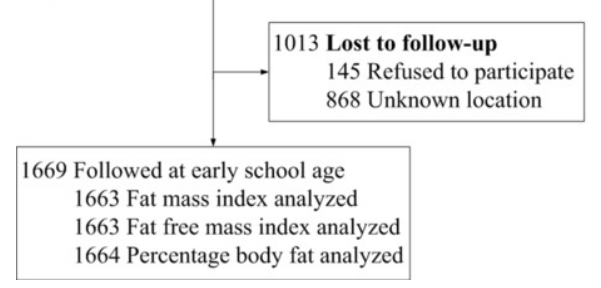

Fig. 1 Flow diagram of recruitment and follow-up in the study cohort.

alt-text: Fig. 1

Table 1 Baseline characteristics of follow-up and lost to follow-up participants. ${ }^{\text {a }}$

alt-text: Table 1

$\begin{array}{ll}\text { Characteristics } & \text { Follow-up }(\mathrm{n}=1669)\end{array}$

$\mathrm{n}$

Maternal age, y

Paternal age, y

Maternal education (primary and below)

Paternal education (primary and below)

Maternal occupation (farmer)

Paternal occupation (farmer)

Household wealth index, median (IQR)

Poorest third

Middle third

Richest third

Primipara

No of male child

Gestation at birth, wk

Preterm (gestational age at birth $<37 w k s$ )

Birth weight, $g$

Low birth weight $(\leq 2500 \mathrm{~g})^{c}$

Small for gestational aged n (\%)/Mean (SD)

24.8 (4.4)

28.0 (4.2)

590 (35.4)

260 (15.6)

1443 (86.9)

$1333(80.1)$

-0.17 (1.87)

581 (34.8)

594 (35.6)

494 (29.6)

1068 (64.0)

1004 (60.2)

39.8 (1.7)

78 (4.7)

3198 (424)

117 (7.1)

277 (16.6)
Lost to follow-up $(\mathrm{n}=1013)$

n (\%)/Mean (SD)

\begin{tabular}{|l|l|}
\hline $24.8(4.3)$ \\
\hline $28.0(4.1)$ \\
\hline $130(31.2)^{\mathrm{b}}$ \\
\hline $870(86.8)$ \\
\hline $786(77.7)$ \\
\hline$-0.23(1.85)$ \\
\hline $360(35.5)$ \\
\hline $341(33.7)$ \\
\hline $312(30.8)$ \\
\hline $645(63.7)$ \\
\hline $583(57.6)$ \\
\hline $39.8(1.7)$ \\
\hline $48(4.7)$ \\
\hline $3201(420)$ \\
\hline $63(6.2)$ \\
\hline $161(15.9)$ \\
\hline
\end{tabular}


a IQR, interquartile range; SD, standard deviation

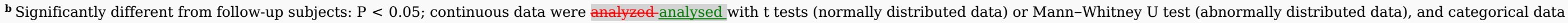
were analyzed-analysed with $\chi^{2}$ tests.

${ }^{\mathbf{c}}$ Assessed because of heaping of birth weight exactly on $2500 \mathrm{~g}$.

d Defined as birth weight below the 10th percentile by gestational age and sex based on international standards.

\subsection{Baseline treatment, birth, and early school aged characteristics}

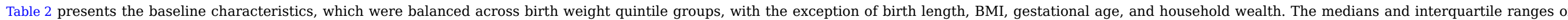
birth weight in each quintile were 2600 (300) g, 3000 (190) g, 3200 (190) g, 3400 (190) g, and 3600 (300) g.

Table 2 Characteristics of the study subjects by birth weight quintiles ${ }^{1}$.

alt-text: Table 2

\begin{tabular}{|c|c|c|c|c|c|c|c|c|c|c|}
\hline & \multicolumn{2}{|r|}{ Q1 $(<2900 \mathrm{~g})$} & \multicolumn{2}{|r|}{ Q2 (2900-3100 g) } & \multicolumn{2}{|c|}{ Q3 $(3100-3300 \mathrm{~g})$} & \multicolumn{2}{|c|}{ Q4 (3300-3500 g) } & \multicolumn{2}{|r|}{ Q5 (>3500 g) } \\
\hline & $\mathrm{n}$ & Mean (SD) & $\mathrm{n}$ & Mean (SD) & $\mathrm{n}$ & Mean (SD) & $\mathrm{n}$ & Mean (SD) & $\mathrm{n}$ & Mean (SD) \\
\hline \multicolumn{11}{|l|}{ Pregnancy } \\
\hline Treatment, n (\%) & 300 & & 350 & & 315 & & 221 & & 483 & \\
\hline Folic acid & & $115(38.3)$ & & $127(36.3)$ & & $105(33.3)$ & & $73(33.0)$ & & $156(32.3)$ \\
\hline Iron + Folic acid & & $84(28.0)$ & & $113(32.3)$ & & $101(32.1)$ & & $82(37.1)$ & & $154(31.9)$ \\
\hline Multiple Micronutrients & & $101(33.7)$ & & $110(31.4)$ & & $109(34.6)$ & & 66 (29.9) & & $173(35.8)$ \\
\hline \multicolumn{11}{|l|}{ Birth } \\
\hline Gender, boys, n (\%) & 300 & $144(48.0)$ & 350 & $181(51.7)$ & 315 & $197(62.5)$ & 221 & $144(65.2)$ & 483 & $338(70.0)$ \\
\hline $\begin{array}{l}\text { Birth weight, g, median } \\
\text { (IQR) }\end{array}$ & 300 & $2600(300)$ & 350 & $3000(190)$ & 315 & $3200(190)$ & 221 & $3400(190)$ & 483 & $3600(300)^{4}$ \\
\hline Birth length, $\mathrm{cm}$ & 260 & $46.8(2.6)$ & 314 & $48.7(2.4)$ & 291 & $49.0(2.0)$ & 205 & $49.5(2.6)$ & 417 & $50.6(2.7)^{4}$ \\
\hline BMI, $\mathrm{kg} / \mathrm{m}^{2}$ & 260 & $11.8(1.3)$ & 314 & $12.6(1.3)$ & 291 & $13.3(1.1)$ & 205 & $13.8(1.5)$ & 417 & $14.5(1.6)^{4}$ \\
\hline Gestation at birth, wk & 300 & $39.3(2.1)$ & 350 & $39.7(1.8)$ & 315 & $39.9(1.4)$ & 221 & $40.0(1.3)$ & 483 & $40.2(1.6)^{4}$ \\
\hline \multicolumn{11}{|l|}{ Early school age } \\
\hline Age, $y$ & 300 & $8.6(0.8)$ & 350 & $8.8(0.8)$ & 315 & $8.7(0.8)$ & 221 & $8.8(0.8)$ & 483 & $8.9(0.8)^{3}$ \\
\hline Household wealth index & 300 & $-0.02(1.01)$ & 350 & $-0.09(0.93)$ & 315 & $0.07(0.98)$ & 221 & $0.21(1.08)$ & 483 & $-0.01(0.99)^{2}$ \\
\hline Maternal BMI, kg/m² & 284 & $21.6(3.2)$ & 333 & $21.5(2.9)$ & 298 & $21.8(2.9)$ & 208 & $22.0(2.6)$ & 465 & $22.0(2.7)$ \\
\hline Paternal BMI, kg/m² & 283 & $22.5(3.0)$ & 332 & $22.4(3.0)$ & 297 & $22.2(2.8)$ & 206 & $22.7(2.8)$ & 465 & $22.2(3.0)$ \\
\hline Dietary pattern, n (\%) & 300 & & 350 & & 315 & & 221 & & 483 & \\
\hline Poor & & $192(64.0)$ & & $228(65.1)$ & & $209(66.3)$ & & $135(61.1)$ & & $301(62.3)$ \\
\hline Balanced & & $31(10.3)$ & & $33(9.4)$ & & $27(8.6)$ & & $24(10.9)$ & & $45(9.3)$ \\
\hline
\end{tabular}




\begin{tabular}{|c|c|c|c|c|c|c|c|c|c|c|}
\hline Rich & & 77 (25.7) & & $89(25.4)$ & & $79(25.1)$ & & $62(28.1)$ & & $137(28.4)$ \\
\hline Physical activities, n (\%) & 289 & & 345 & & 306 & & 217 & & 472 & \\
\hline Often & & $177(61.2)$ & & $200(58.0)$ & & $168(54.9)$ & & $127(58.5)$ & & $270(57.2)$ \\
\hline Occasionally & & 112 (38.8) & & $145(42.0)$ & & $138(45.1)$ & & $90(41.5)$ & & $202(42.8)$ \\
\hline
\end{tabular}

${ }^{1} \mathrm{IQR}$, interquartile range; BMI, body mass index = body mass $(\mathrm{kg}) /$ length or height $(\mathrm{m})^{2}$.

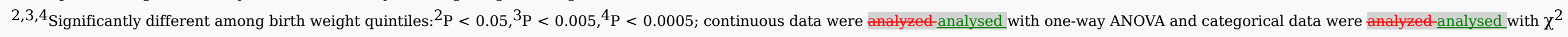
tests.

\subsection{Birth weight and early school aged body size and composition}

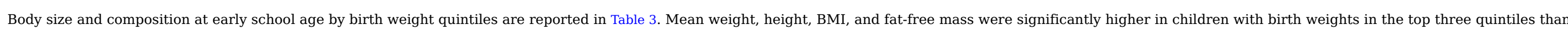

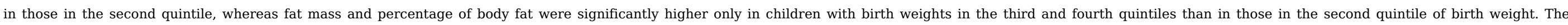
incidence of overweight/obesity was significantly higher in children in the upper three quintiles of birth weight when than in children in the second quintile of birth weight.

Table 3 Body size and composition of the study subjects by birth weight quintiles ${ }^{1}$.

alt-text: Table 3

\begin{tabular}{|c|c|c|c|c|c|c|c|c|c|c|}
\hline & \multicolumn{2}{|r|}{ Q1 (<2900 g) } & \multicolumn{2}{|c|}{ Q2 $(2900-3100 \mathrm{~g})$} & \multicolumn{2}{|r|}{ Q3 (3100-3300 g) } & \multicolumn{2}{|r|}{ Q4 (3300-3500 g) } & \multicolumn{2}{|r|}{ Q5 (>3500 g) } \\
\hline & $\mathrm{n}$ & Mean (SD) & $\mathrm{n}$ & Mean (SD) & $\mathrm{n}$ & Mean (SD) & $\mathrm{n}$ & Mean (SD) & $\mathrm{n}$ & Mean (SD) \\
\hline Weight, kg & 299 & 23.5 & 347 & $24.9(3.9)$ & 315 & $26.0(4.8)$ & 221 & $26.5(4.3)$ & 483 & $26.8(4.6)^{4}$ \\
\hline WAZ $^{5}$ & 283 & $-0.96(0.95)$ & 324 & $-0.74(0.86)$ & 302 & $-0.43(0.97)$ & 210 & $-0.31(0.92)$ & 438 & $-0.32(0.91)^{4}$ \\
\hline Height, $\mathrm{cm}$ & 299 & $125.8(6.1)$ & 348 & $128.1(6.4)$ & 315 & $129.0(6.3)$ & 221 & $129.7(6.6)$ & 483 & $130.7(6.6)^{4}$ \\
\hline HAZ & 299 & $-0.76(0.93)$ & 348 & $-0.53(0.84)$ & 315 & $-0.32(0.80)$ & 221 & $-0.26(0.89)$ & 483 & $-0.16(0.86)^{4}$ \\
\hline BMI, $\mathrm{kg} / \mathrm{m}^{2}$ & 298 & $14.8(1.4)$ & 347 & $15.1(1.5)$ & 315 & $15.6(1.9)$ & 221 & $15.7(1.6)$ & 483 & $15.6(1.6)^{4}$ \\
\hline BAZ & 298 & $-0.85(0.98)$ & 347 & $-0.67(0.92)$ & 315 & $-0.40(1.05)$ & 221 & $-0.31(0.98)$ & 483 & $-0.40(0.98)^{4}$ \\
\hline FM, kg, median (IQR) & 298 & $2.2(1.6)$ & 347 & $2.7(1.6)$ & 315 & $2.9(1.9)$ & 221 & $3.0(2.2)$ & 482 & $2.8(2.1)^{4}$ \\
\hline FFM, kg, median (IQR) & 298 & $20.6(3.9)$ & 347 & $21.8(3.8)$ & 315 & $22.6(4.2)$ & 221 & $23.0(3.7)$ & 482 & $23.3(4.4)^{4}$ \\
\hline FMI, kg/m², median (IQR) & 298 & $1.4(0.9)$ & 347 & $1.7(1.0)$ & 315 & $1.7(1.1)$ & 221 & $1.8(1.4)$ & 482 & $1.7(1.2)^{4}$ \\
\hline FFMI, kg/m², median (IQR) & 298 & $13.2(1.1)$ & 347 & $13.4(1.1)$ & 315 & $13.6(1.1)$ & 221 & $13.7(1.0)$ & 482 & $13.8(0.9)^{4}$ \\
\hline PBF, \%, median (IQR) & 298 & $9.8(5.5)$ & 347 & $11.1(5.9)$ & 315 & $11.3(6.4)$ & 221 & $11.7(7.0)$ & 482 & $11.0(7.0)^{3}$ \\
\hline Overweight/obesity, n (\%)6 & 298 & $11(3.7)$ & 347 & $12(3.5)$ & 315 & $32(10.2)$ & 221 & $17(7.7)$ & 482 & $35(7.3)^{2}$ \\
\hline
\end{tabular}

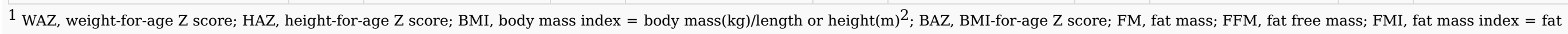
$\operatorname{mass}(\mathrm{kg}) / \mathrm{height}(\mathrm{m})^{2} ; \mathrm{FFMI}$, fat free mass index $=$ fat free mass $(\mathrm{kg}) /$ height $(\mathrm{m})^{2} ;$ PBF: percentage of body fat $=$ fat mass $(\mathrm{kg}) / \mathrm{total}$ body mass $(\mathrm{kg}) \times 100 ; \mathrm{SD}$, standard deviation.

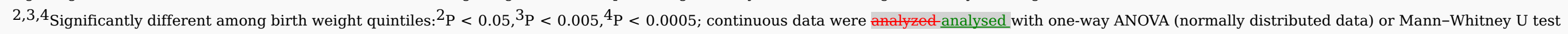
(abnormally distributed data), and categorical data were analyzed-analysed with $\chi^{2}$ tests.

$5 n=1557$ because the database for weight SDS was not available for the age of $10 y$. 
${ }^{6}$ Overweight was defined as PBF between 85th and 95th percentiles of each age and sex group and obesity was defined as PBF above 95th percentile of each age and sex group.

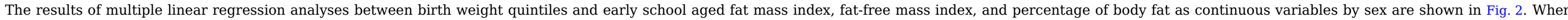

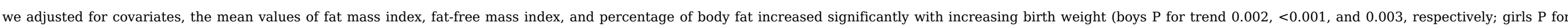

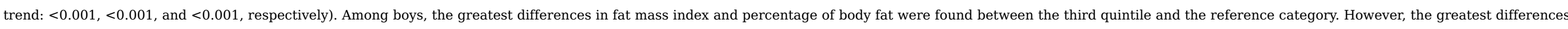

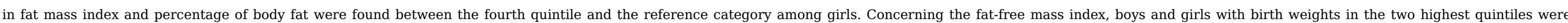
$0.23 \mathrm{~kg} / \mathrm{m}^{2}$ and $0.25 \mathrm{~kg} / \mathrm{m}^{2}$ heavier than those with birth weights in the second quintile, respectively.
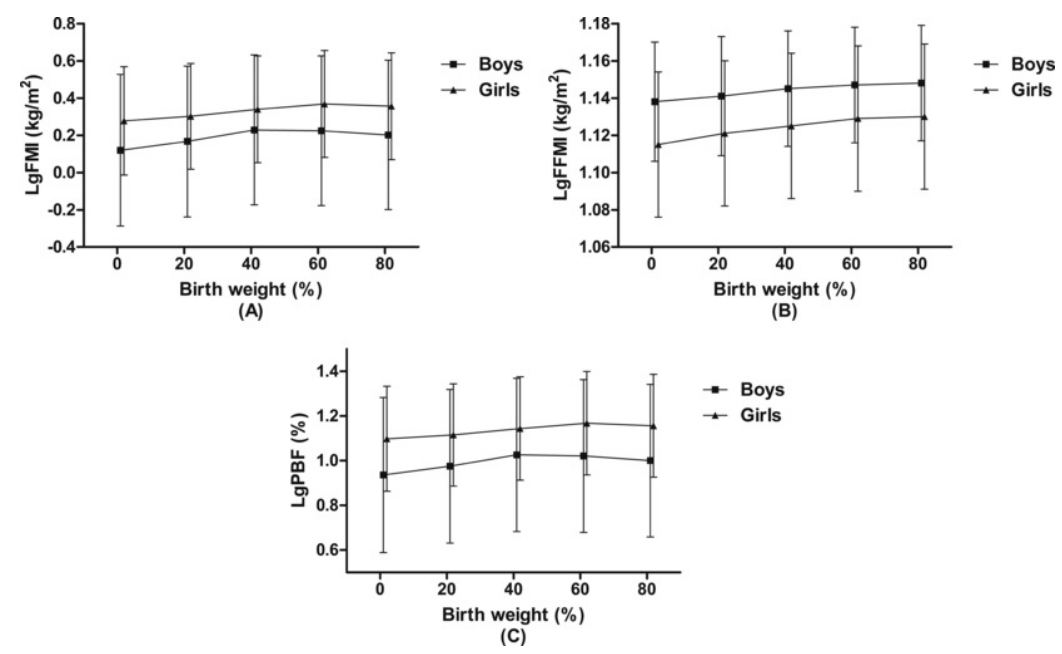

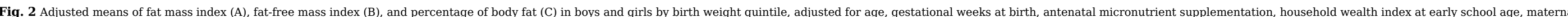
BMI and paternal BMI. Error bars are 95\% CIs.

\section{alt-text: Fig. 2}

\subsection{Birth weight and early school aged overweight/obesity}

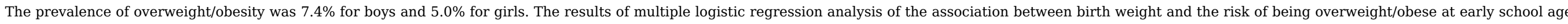

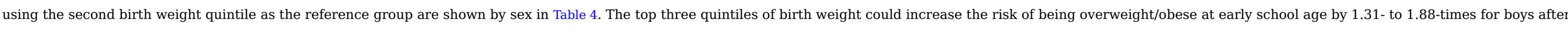

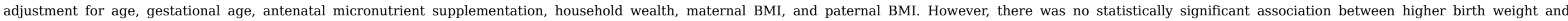

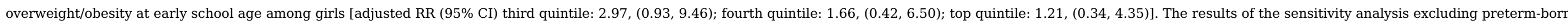
children (data not shown) showed similar associations.

Table 4 Risk ratios of overweight/obesity at early school age by birth weight quintiles. ${ }^{\text {a }}$

alt-text: Table 4

Birth weight Number of participants N N N N $\quad$ Number of case

Model 1

Model 2

\begin{tabular}{|l|l|}
\hline Q1 & 143 \\
\hline Q2 & 179 \\
\hline Q3 & 197 \\
\hline
\end{tabular}

3.40

RR

$95 \%, 10.29,3.36)$
[Reference]

$95 \% \mathrm{CI}$

$(1.34,8.64)$
$\mathrm{RR}$ $(0.17,2.47)$ [Reference]

(1.13, 7.32) 


\begin{tabular}{|c|c|c|c|c|c|c|c|}
\hline & Q4 & 144 & 13 & 2.81 & $(1.04,7.58)$ & 2.40 & $(0.87,6.66)$ \\
\hline & Q5 & 337 & 29 & 2.75 & $(1.12,6.77)$ & 2.31 & $(0.92,5.80)$ \\
\hline \multirow[t]{5}{*}{ Girls } & Q1 & 156 & 6 & 1.10 & $(0.34,3.52)$ & 1.55 & $(0.43,5.57)$ \\
\hline & Q2 & 168 & 6 & 1 & [Reference] & 1 & [Reference] \\
\hline & Q3 & 118 & 11 & 2.81 & $(0.99,7.93)$ & 2.80 & $(0.86,9.06)$ \\
\hline & Q4 & 77 & 4 & 1.46 & $(0.40,5.34)$ & 1.66 & $(0.43,6.41)$ \\
\hline & Q5 & 145 & 6 & 1.15 & $(0.37,3.59)$ & 1.18 & $(0.34,4.08)$ \\
\hline
\end{tabular}

Model 1: Adjusted for age only.

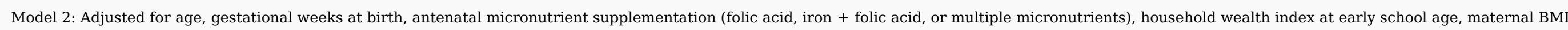
and paternal BMI.

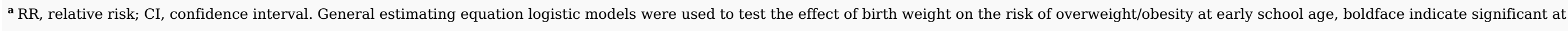

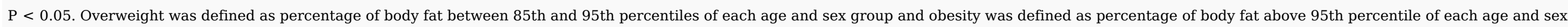
group.

\section{Discussion}

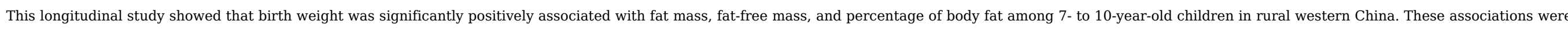

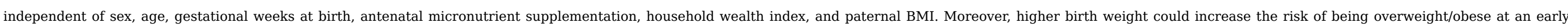
school age among boys.

\subsection{Interpretation of the findings and comparison with other studies}

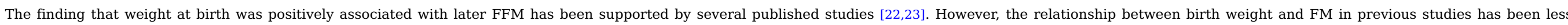

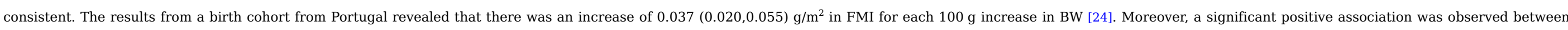

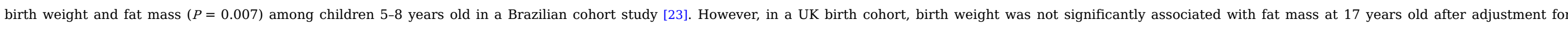

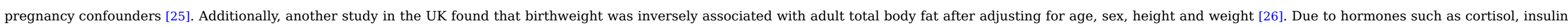

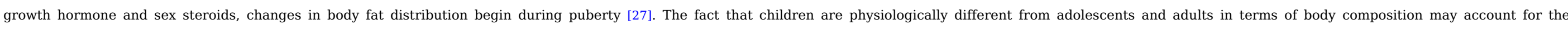
inconsistent results from studies that targeted children, adolescents or adults.

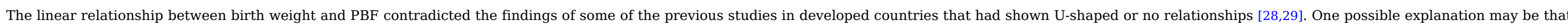
body fat percent is ethnicity-specific, which results from the differences in relative leg length, frame size and physical activity level [30-32].

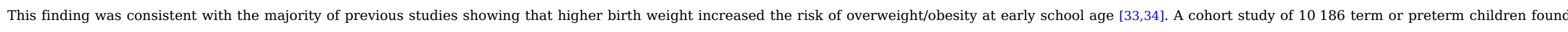

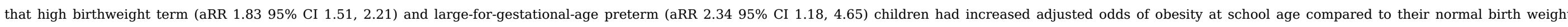

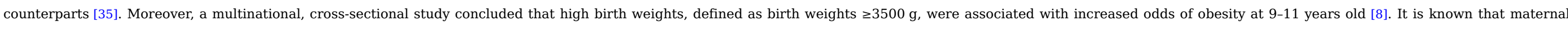

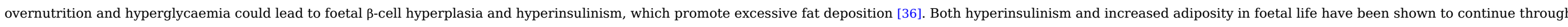

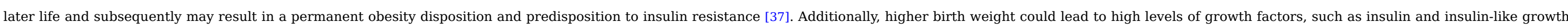

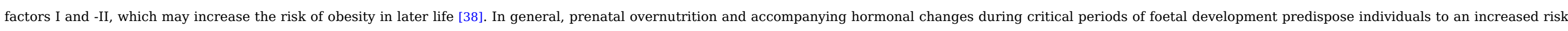
of obesity over the long term.

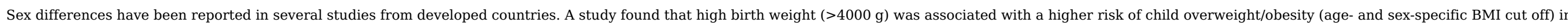




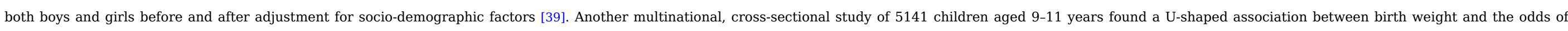

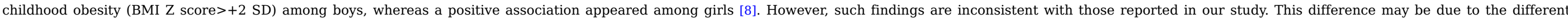
categories of birth weight and various definitions of obesity. More longitudinal studies are needed to assess whether the association between birth weight and childhood obesity differs by sex.

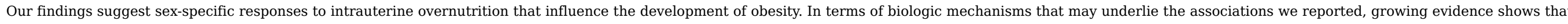

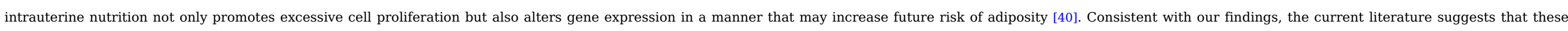

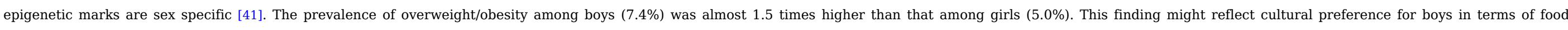
allocation [42] and caretaking [43].

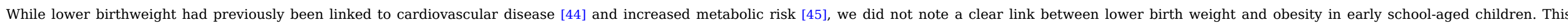

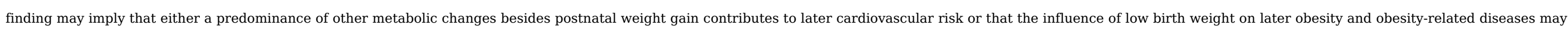
occur after the age of 10 years.

\subsection{Strengths and limitations}

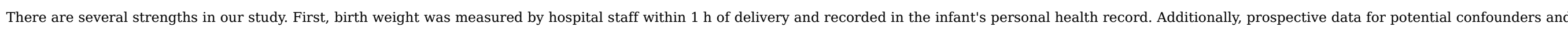

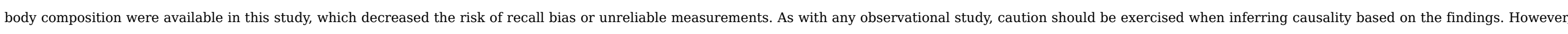
our findings were consistent before and after adjustments for potential confounding by most known variables that were plausibly associated with birth weight and childhood body composition.

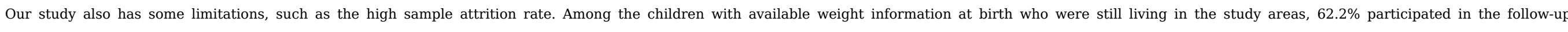

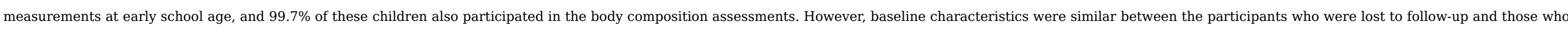

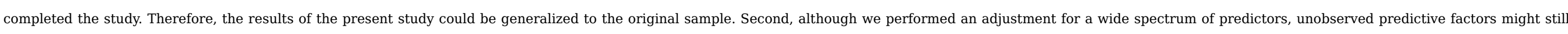

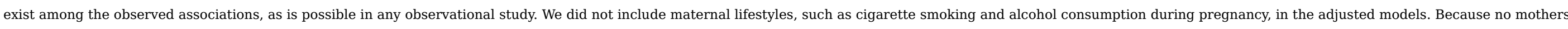

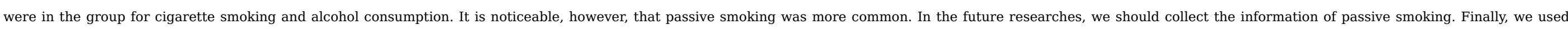

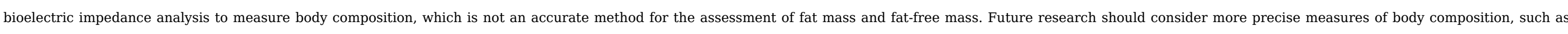
dual-energy X-ray absorptiometry scans or air displacement methods, to allow for a better estimate of the true strength of these associations.

\section{Conclusions}

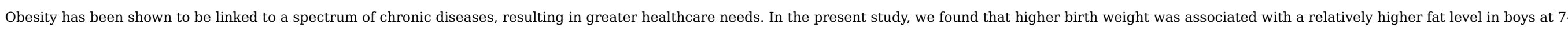

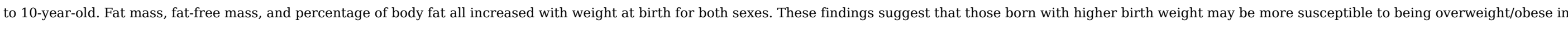
later life. Regulation of pre-pregnant and pregnant nutritional status and diet may be useful to prevent childhood obesity and obesity-related health problems by minimizing the risk of foetal overgrowth.

\section{Funding}

This study was funded by UNICEF and the National Natural Science Foundation of China (grant number 81872633).

\section{Author contributions}

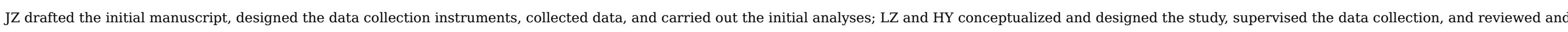

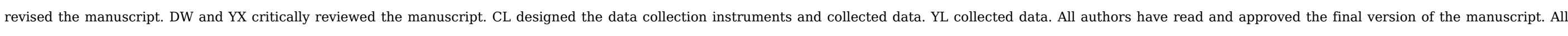
authors agreed to be accountable for all aspects of the work.

\section{Conflict of interest}

The authors declare no conflicts of interest. 


\section{Acknowledgements}

We thank the entire team, including the field managers, interviewers, and technicians, and the study area coordinators for their help with the field procedures.

\section{References}

[1] T. Lobstein, R. Jackson-Leach, M.L. Moodie, K.D. Hall, S.L. Gortmaker, B.A. Swinburn, et al., Child and adolescent obesity: part of a bigger picture, Lancet 385, 2015, 2510-2520.

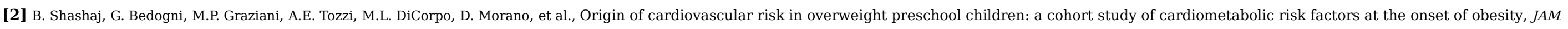
Pediatrics 168, 2014, 917-924.

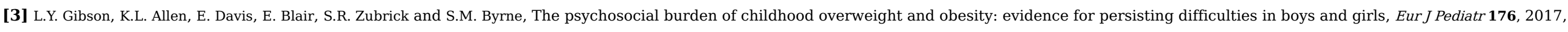
925-933.

[4] S.E. Litwin, Childhood obesity and adulthood cardiovascular disease: quantifying the lifetime cumulative burden of cardiovascular risk factors, J Am Coll Cardiol 64, 2014, 1588-1590.

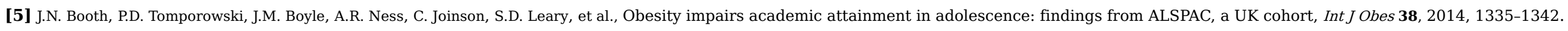

[6] W.H. Dietz, Periods of risk in childhood for the development of adult obesity - what do we need to learn?, J Nutr 127, 1997, 1884-1886.

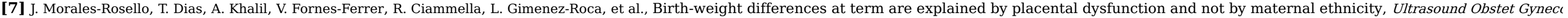
52, 2018, 488-493.

[8] Y. Qiao, J. Ma, Y. Wang, W. Li, P.T. Katzmarzyk, J.P. Chaput, et al., Birth weight and childhood obesity: a 12-country study, Int J Obes Suppl 5, 2015 , 74-79.

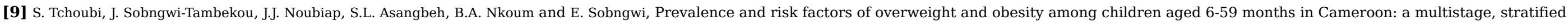
cluster sampling nationwide survey, PLoS One 10, 2015, e0143215.

[10] N. Li, E. Liu, S. Sun, J. Guo, L. Pan, P. Wang, et al., Birth weight and overweight or obesity risk in children under 3 years in China, Am J Hum Biol 26, 2014 , 331-336.

[11] J.C. Wells and T.J. Cole, Adjustment of fat-free mass and fat mass for height in children aged 8 y, Int J Obes Relat Metab Disord 26, 2002, 947-952.

[12] J.C. Wells and M.S. Fewtrell, Measuring body composition, Arch Dis Child 91, 2006, 612-617.

[13] P. Sonksen, Determination and regulation of body composition in elite athletes, BrJ Sports Med 52, 2018, 219-229.

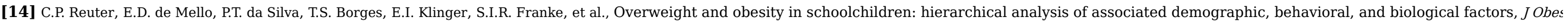
61, 2018, 28-34.

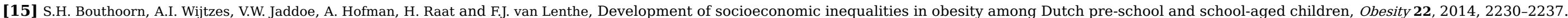

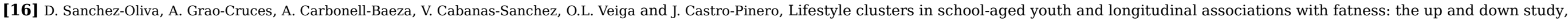
J Pediatr 203, 2018, 317-324.

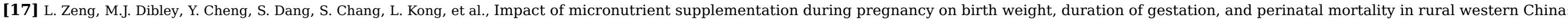
double blind cluster randomised controlled trial, BMJ 337, 2008, a2001.

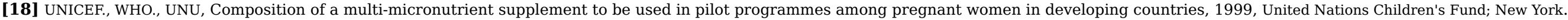

[19] L. Zhu, R. Zhang, S. Zhang, W. Shi, W. Yan, X. Wang, et al., Chinese neonatal birth weight curve for different gestational age, Chin J Pediatr 53, $2015,97-103$.

[20] WHO, Growth reference data for 5-19 years, 2007. 
[21] H.D. McCarthy, T.J. Cole, T. Fry, S.A. Jebb and A.M. Prentice, Body fat reference curves for children, Int J Obes 30, 2006, 598-602.

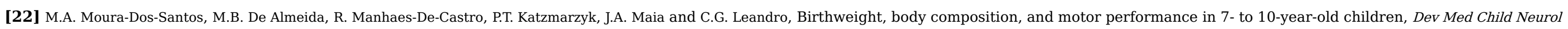
57, 2015, 470-475.

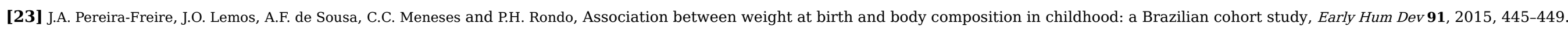

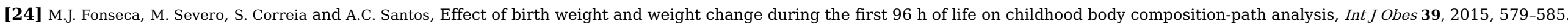

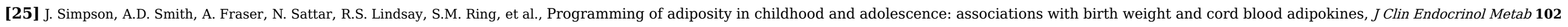
2017, 499-506.

[26] C.R. Gale, C.N. Martyn, S. Kellingray, R. Eastell and C. Cooper, Intrauterine programming of adult body composition, J Clin Endocrinol Metab 86, $2001,267-272$.

[27] J.N. Roemmich and A.D. Rogol, Hormonal changes during puberty and their relationship to fat distribution, Am J Hum Biol 11, $1999,209-224$.

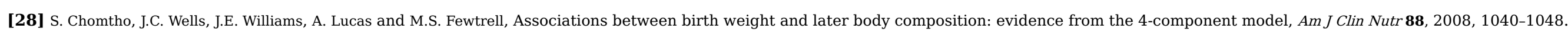

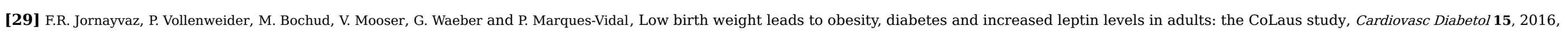
73-82.

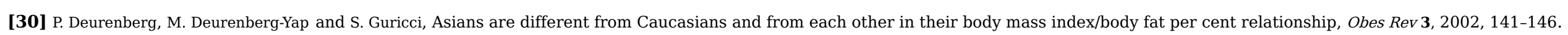

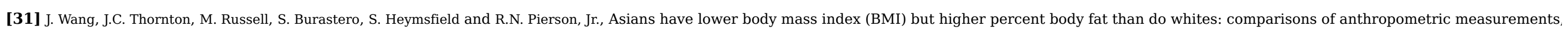
Am J Clin Nutr 60, 1994, 23-28.

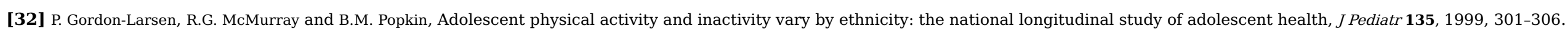

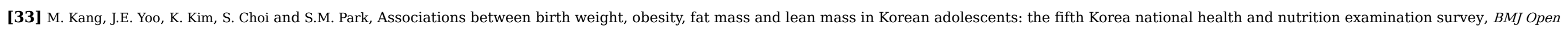
8, 2018, e018039.

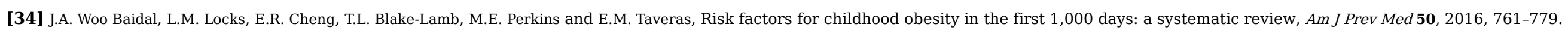

[35] N. Kapral, S.E. Miller, R.J. Scharf, M.J. Gurka and M.D. DeBoer, Associations between birthweight and overweight and obesity in school-age children, Pediatr Obes 13, 2018, 333-341.

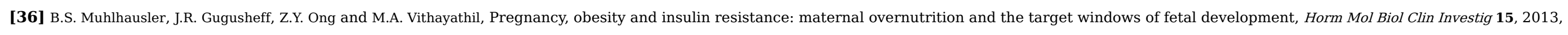
$25-36$.

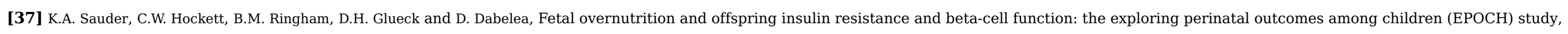
Diabet Med 34, 2017, 1392-1399.

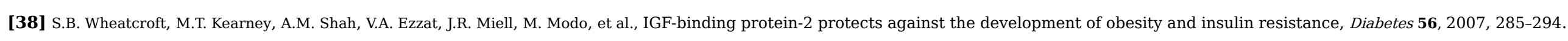

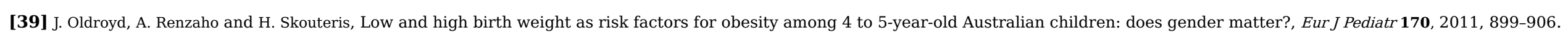

[40] R. Saffery and B. Novakovic, Epigenetics as the mediator of fetal programming of adult onset disease: what is the evidence?, Acta Obstet Gynecol Scand $\mathbf{9 3}$, 2014, 1090-1098.

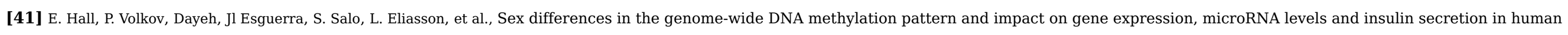
pancreatic islets, Genome Biol 15, 2014, 522-543.

[42] M.J. Graham, U. Larsen and X. Xu, Son preference in Anhui province, China, Int Fam Plan Perspect 24, 1998, $72-77$. 
[43] E. Susan, F. Zhai, S. Xu and M. Yang, China's one-child policy and the care of children: an analysis of qualitative and quantitative data, Soc Forces 79, 2001 , 913-943.

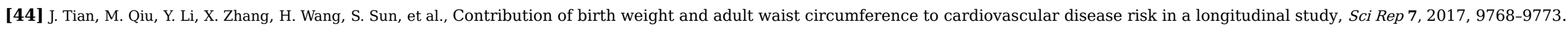

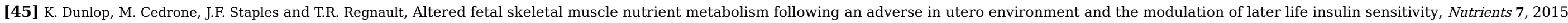
1202-1216.

\section{Queries and Answers}

Query: Please check Research registration in Abstract section, and correct if necessary.

Answer: it's right

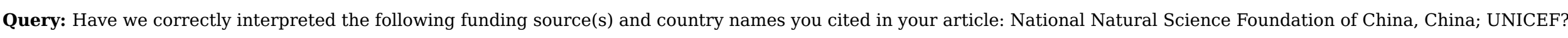
Answer: Yes

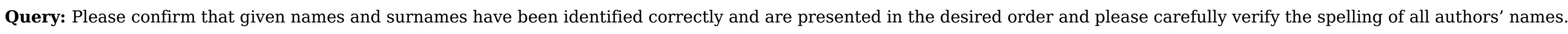
Answer: Yes

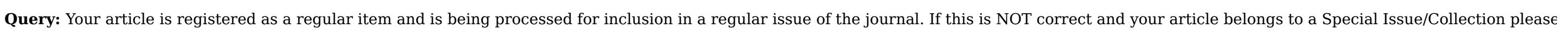
contact o.pyne@elsevier.com immediately prior to returning your corrections.

Answer: Yes 